\title{
SuperTIGER and the Origin of Galactic Cosmic-Rays
}

\author{
T. HAMS ${ }^{1,6}$, W. R. BINNS ${ }^{2}$, R. G. BOSE ${ }^{2}$, D. L. BRAUN ${ }^{2}$, T. J. BRANDT ${ }^{1}$, \\ W. M. DANIELS ${ }^{1}$, P. F. DOWKONTT ${ }^{2}$, S. P. FITZSIMMONS ${ }^{1}$, D. J. HAHNE ${ }^{1}$, \\ M. H. ISRAEL ${ }^{2}$, J. KLEMIC ${ }^{3}$, A. W. LABRADOR ${ }^{3}$, J. T. LINK $^{1,6}$, R. A. MEWALDT ${ }^{3}$, \\ J. W. MITCHELL ${ }^{1}$, P. MOORE ${ }^{2}$, R. P. MURPHY ${ }^{2}$, M. A. OLEVITCH ${ }^{2}$, B. F. RAUCH ${ }^{2}$, \\ K. SAKAI ${ }^{1,6}$, F. SAN SEBASTIAN ${ }^{1}$, M. SASAKI ${ }^{* 1,6}$, G. E. SIMBURGER ${ }^{2}$, E. C. STONE ${ }^{3}$, \\ C. J. WADDINGTON ${ }^{4}$, J. E. WARD ${ }^{2}$ M. E. WIEDENBECK ${ }^{5}$
}

${ }^{1}$ NASA Goddard Space Flight Center, Greenbelt, MD 20771, USA

${ }^{2}$ Washington University, St. Louis, MO 63130, USA

${ }^{3}$ California Institute of Technology, Pasadena, CA 91125, USA

${ }^{4}$ University of Minnesota, Minneapolis, MN 55455, USA

5 Jet Propulsion Laboratory, California Institute of Technology, Pasadena, CA 91109, USA

${ }^{6}$ Center for Research and Exploration in Space Science and Technology (CRESST)

\begin{abstract}
The SuperTIGER (Super Trans-Iron Galactic Element Recorder) long-duration balloon instrument has measured the abundances of galactic cosmic-ray elements from ${ }_{10} \mathrm{Ne}$ to ${ }_{40} \mathrm{Zr}$ with high statistics and single element resolution and its measurements extend to about ${ }_{60} \mathrm{Nd}$. SuperTIGER is the first instrument to measure the abundance of every element from $\mathrm{Z}=30$ to 40 with significant statistics, recording more than 600 nuclei with atomic number $\mathrm{Z}>30$ in its first flight. Its measured nuclear charge resolution is excellent, with $\sigma_{Z}=0.16$ c.u. at ${ }_{26} \mathrm{Fe}$. From 0.8 to $10 \mathrm{GeV} /$ nucleon it also measures the energy spectra of the more abundant elements with $10 \leq \mathrm{Z} \leq 30$. SuperTIGER-1 launched from Williams Field, McMurdo Station, Antarctica, on December 8, 2012, flew for a record 55 days and over 2.5 revolutions around the continent, returned data on over 50 million heavy cosmic ray nuclei. The instrument has now been recovered from Antarctica and preparations are underway for its next flight. Instrument and flight details, methods of charge identification employed, preliminary results from the SuperTIGER-1 balloon flight, and a summary of the recovery will be presented. The SuperTIGER-1 measurements will be discussed in the context of their stringent tests of the OB association model for the origin of galactic cosmic rays. Finally, planned improvements to the SuperTIGER instrument and future flight plans will be described. Treatment of the data depends somewhat on $\mathrm{Z}$ and details of the data analysis and results in ranges $10 \leq \mathrm{Z} \leq 29$ and $30 \leq \mathrm{Z} \leq 40$ are given in other talks at this conference. SuperTIGER was developed by Washington University in St. Louis, NASA Goddard Space Flight Center, the California Institute of Technology, Jet Propulsion Laboratory, and the University of Minnesota.
\end{abstract}

The 34th International Cosmic Ray Conference,

30 July- 6 August, 2015

The Hague, The Netherlands 


\section{Introduction}

SuperTIGER is a large-area instrument designed to make precision measurements of the elemental composition of ultra-heavy galactic cosmic rays (UHGCR) with atomic number $\mathrm{Z} \geq$ 30 that is built on the heritage of the Trans-Iron Galactic Element Recorder (TIGER), which was successfully flown on two balloons launched in Antarctica in December of 2001 and 2003 [四]. The principal objective of the SuperTIGER program is to measure the abundances of nuclei with $30 \leq \mathrm{Z}$ $\leq 42$ with clear individual element resolution and high statistical precision. A secondary objective is to accurately measure the energy spectra of the more abundant light elements with $12 \leq \mathrm{Z} \leq$ 28. The abundance measurements provide sensitive tests and clarification of the OB-association model of Galactic cosmic-ray origins, and will test models for atomic processes by which nuclei are selected for acceleration to cosmic ray energies. The cosmic-ray source is understood as a mixture of old interstellar material and fresh material from the outflow of massive stars (importantly including Wolf-Rayet stars and their precursor phases) and ejecta from core-collapse supernovae (SNII, SNIb,c), which occur mostly in OB associations. This model is supported by the ${ }^{22} \mathrm{Ne} /{ }^{20} \mathrm{Ne}$ and ${ }^{58} \mathrm{Fe} /{ }^{56} \mathrm{Fe}$ overabundances in the GCRs measured most recently by the CRIS instrument on ACE as well as the element ratio N/O [ [ $[$, B]]. In addition, elements that reside primarily in grains in interstellar space (refractory elements) are preferentially accelerated compared to those that reside primarily as gas (volatile elements), and this enrichment appears to be mass dependent for both refractories and volatiles. The mass-dependent element enrichment is supported by abundances measured by TIGER at GeV/nuc energies [四], ACE at energies of hundreds of MeV/nuc [䧃], and $\mathrm{CREAM}$ at TeV/nuc energies [[]]. Both isotopic and elemental abundances point to a cosmic-ray source with $\sim 80 \%$ of the mass having the composition characteristic of the material of our Solar System (SS), and $\sim 20 \%$ having a composition characteristic of outflow and ejecta from massive stars (MSP-massive star production). In this picture, measurements of the GCR source composition sample the composition of $\mathrm{OB}$ associations in our Galaxy, and measurement of the rare $\mathrm{Z} \geq$ 30 elements allows us to probe these regions for enrichments expected from nucleosynthesis in massive stars. Analysis of data from our recent SuperTIGER flight will provide an improved test of this model. Details of these analyses are discussed in [6, $\square]$.

\section{SuperTIGER-1 Flight}

\subsection{Instrument}

SuperTIGER consists of two identical modules, each comprised of scintillating fiber hodoscopes (H1, H2), three scintillator detectors (S1, S2, and S3), an aerogel Cherenkov detector and an acrylic Cherenkov detector (C0, C1) [8] (Fig., [1). The hodoscope determines the trajectory of incident particles. The scintillators and Cherenkov detectors determine the particle charge and energy.

The hodoscopes use a coded readout scheme originally developed for TIGER to limit the number of PMTs needed. Each hodoscope plane uses two orthogonal layers of square scintillating

\footnotetext{
* Speaker.

†E-mail: Makoto.Sasaki@nasa.gov

†Now at Institut de Fisica d’Altes Energies (IFAE), Ballaterra, Spain
} 


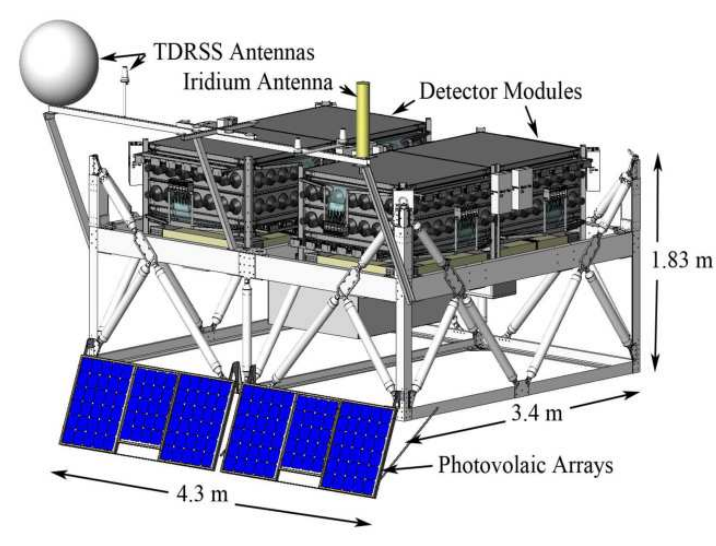

Figure 1: The SuperTIGER instrument.

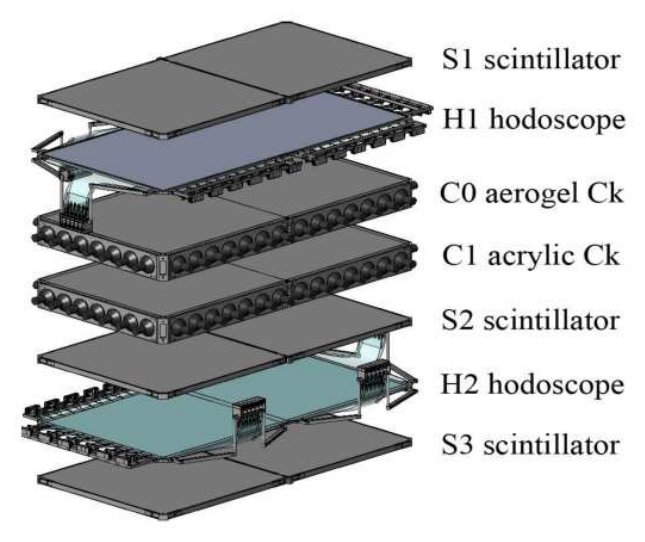

Figure 2: Single module shown in expanded view.

fibers covering a fiducial area of $2.4 \mathrm{~m} \times 1.16 \mathrm{~m}$. Long-axis fibers are $1.4 \mathrm{~mm}$ square while short fibers are $1 \mathrm{~mm}$. The fibers are formatted into "tabs" of 6 (long) or 8 (short) fibers, so segmentation is $\sim 8 \mathrm{~mm}$. Groups of 12 adjacent tabs at one end of each layer are viewed by a single Hammamatsu R1924A PMT to give coarse spatial resolution. The tabs on the opposite ends of the fibers are sequentially routed to PMTs so each tab within a coarse group is viewed by a different PMT. This acts as a "vernier" for single-tab localization. A good event has a hit at both coarse and fine ends, so the uncertainty in position is $\sigma_{r m s}=8 \mathrm{~mm} / \sqrt{12}=2.3 \mathrm{~mm}$. Averaged over the effective opening angle of the instrument, for $\mathrm{Z}=40$ the resulting uncertainty in the secant correction contributes only $\sigma_{t r a j}=0.003$ c.u. to $\sigma_{Z}$. The PMT signals are pulse-height analyzed so that large signals from heavy nuclei can be readily distinguished from knock-on electrons.

Each half-module scintillation detector uses a sheet of $1.16 \mathrm{~m} \times 1.16 \mathrm{~m} \times 1 \mathrm{~cm}$ ELJEN Technology EJ-208B plastic scintillator. The scintillation light produced is carried to the edges of the sheet by total internal reflection and is coupled through a thin air gap to four EJ-280 wavelengthshifter (WLS) bars around its perimeter. Each WLS bar is read out by Hamamatsu R1924A 2.54 $\mathrm{cm}$ diameter PMTs coupled to both ends using optical epoxy. Two such detector units were used in each module layer to provide both $\mathrm{dE} / \mathrm{dx}$ measurements and the event trigger. The scintillators were the largest sheets that could be produced with the required uniformity. The scintillation detector enclosures were floored with an ultra-low density Al-foam composite developed at GSFC. This Al-foam composite was also used to support the hodoscopes and as floors for the Cherenkov enclosures. The tops of both scintillation detector and Cherenkov detector enclosures were 0.1 $\mathrm{mm}$ hard $\mathrm{Al}$ foil. Overall the use of the foil and $\mathrm{Al} /$ foam composites reduced interactions by $\sim 20 \%$ compared to the materials used in TIGER. The scintillators sit on thin layers of Depron foam. Sheets of highly reflective aluminized mylar above and below the scintillators decouple the internal reflection surfaces from the foam and improve light collection and uniformity. The PMT bases used radically tapered voltage dividers providing nearly linear response to $2 \times 10^{5}$ photoelectrons (p.e.) and an effective dynamic range of $2 \times 10^{4}$ to cover the charge range $10 \leq \mathrm{Z} \leq$ 60 , including the variations in light reaching each PMT due to particle incident position and angle. The bases incorporated charge-sensitive preamplifiers (CSA) to eliminate pick-up noise that might 
reduce the resolution of the measurement. The PMTs were wrapped with a single $0.1 \mathrm{~mm}$ thick layer of mu-metal foil to shield the varying effects of the Earth's magnetic field with instrument location and orientation.

The Cherenkov detectors for SuperTIGER were based on the extensive experience of the collaboration with the IMAX, ISOMAX, TIGER, BESS, HEAO-3 and BESS-Polar silica-aerogel and acrylic Cherenkov detectors. Light integration volumes lined with highly reflective GORE DRP Light-Reflective Material of $0.25 \mathrm{~mm}$ thickness collect the Cherenkov light produced by the silica-aerogel (C0) and acrylic (C1) radiators. Each optical volume has an aperture of $118 \mathrm{~cm}$ x 240 $\mathrm{cm}$ and is $20 \mathrm{~cm}$ tall. The enclosures are divided into two half-module structures for recovery, but these are open at one end and so create a unified optical system. Each detector is read out by 4212.7 cm Hamamatsu R877-100 PMTs with "Super-Bialkali" photocathodes for high quantum efficiency. Each C0 module contains eight aerogel blocks, each approximately $55 \mathrm{~cm} \times 55 \mathrm{~cm}$ and $3 \mathrm{~cm}$ tall. Three of the four half-modules contain aerogel blocks with $\mathrm{n}=1.043$ (12 blocks total), while one half-module contains four blocks with $\mathrm{n}=1.025$. These have thresholds of $2.5 \mathrm{GeV} /$ nucleon and $3.3 \mathrm{GeV} /$ nucleon, respectively. The aerogel blocks are mounted on thin composite "pallets" covered in GORE DRP and are held in position by a layer of polyethylene terephthalate, similar to "Saran" wrap, using a technique adapted from BESS/BESS-Polar. C1 uses acrylic radiators with bis-MSB wavelength shifter added that were cast for SuperTIGER by Polycast. This material has an index of refraction of 1.49 , corresponding to a threshold of $0.35 \mathrm{GeV} /$ nucleon. In each module the radiator consists of two $1.16 \mathrm{~m}$ x $1.16 \mathrm{~m}$ sheets meeting near the midline of the detector. The Cherenkov detector PMTs were mounted in custom hydroformed $0.3 \mathrm{~mm}$ thick mu-metal sleeves that served as magnetic shields, mechanical support and light close-out. Their bases used voltage dividers that were even more radically tapered than those for the scintillators, and which could measure from 10 to $\sim 2 \times 10^{5}$ pe (dynamic range of $2 \times 10^{4}$ ) with only $2 \%$ non-linearity in order to span the $10 \leq \mathrm{Z} \leq 60$ range. As with the scintillators, the voltage dividers incorporated integrated CSAs.

\subsection{Flight}

SuperTIGER-1 was launched on Dec. 8, 2012 (UTC), from Williams Field, Antarctica. In about 2.7 circumnavigations of Antarctica, it flew for over 55 days at altitudes from about $35.2 \mathrm{~km}$ to $39.7 \mathrm{~km}$ (Fig. [1), which correspond to $\sim 6.4$ to $3.5 \mathrm{~g} / \mathrm{cm}^{2}$ of residual atmosphere respectively. The flight was terminated on Feb. 1, 2013 due to concerns about increasing instability in the highaltitude winds and safety margins in a CSBF balloon termination battery which was rated for 60 days with $10 \%$ margin. Termination was carried out "over-the-horizon" at a location on the West Antarctic Ice Sheet (WAIS) with $82^{\circ} 14^{\prime} 44.40^{\prime \prime}$ South latitude and $81^{\circ} 54^{\prime} 40.80^{\prime \prime}$ West longitude, $1625 \mathrm{~km}$ from McMurdo and $600 \mathrm{~km}$ from the nearest long-term US camp (WAIS Divide). The flight trajectory is shown in Fig. $⿴$. The flight set duration records for heavy scientific payloads and for heavy-lift scientific balloons.

The instrument returned excellent data on over $67 \times 10^{6}$ cosmic-ray nuclei above the trigger threshold at $\sim Z \geq 10$. Nearly all high-priority data $(Z \geq 22)$ could be sent through TDRSS when satellite view angles supported transmission rates as low as 20kbs. Large fractions of lower priority data ( $10 \leq \mathrm{Z} \leq 22$ ) were also returned. Overall, considering outages and very-low-rate periods, TDRSS data transmission was about $80 \%$ efficient, giving the equivalent of 44 days of 


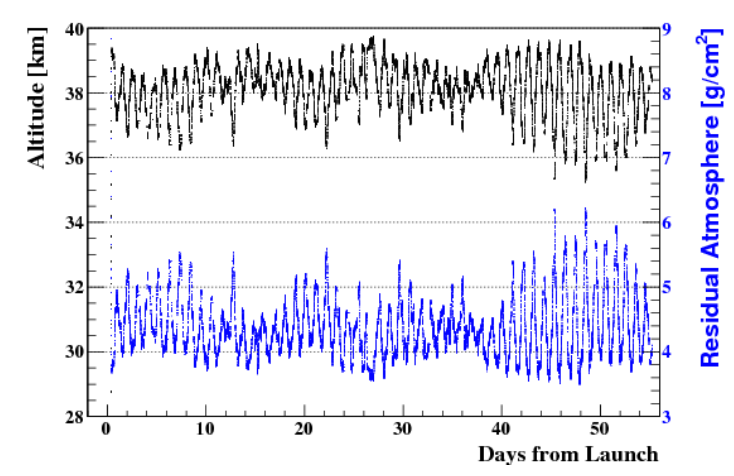

Figure 3: Flight altitude and residual atmosphere of the SuperTIGER-1. The float altitude is from about $35.2 \mathrm{~km}$ to $39.7 \mathrm{~km}$ (black line) and the residual atmosphere is from $3.5 \mathrm{~g} / \mathrm{cm}^{2}$ to $6.4 \mathrm{~g} / \mathrm{cm}^{2}$ (blue line).

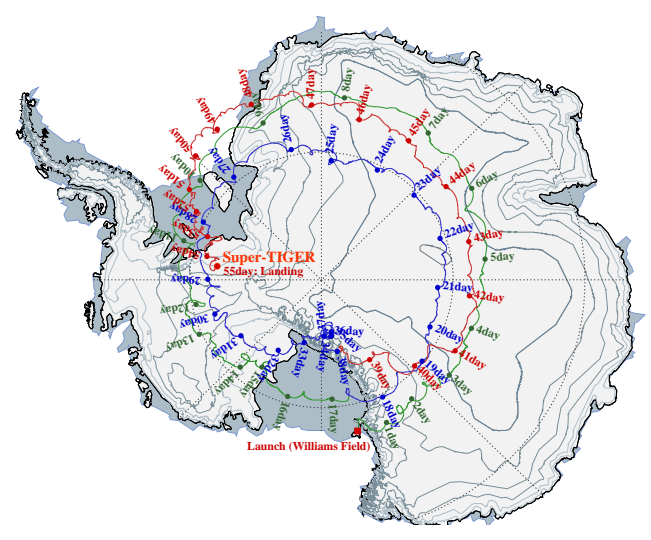

Figure 4: Flight trajectories of SuperTIGER1. Green line indicates the first revolution, blue line indicates the second revolution, and red line indicates the third revolution.

data recorded for high $\mathrm{Z}$ events. All triggered events occurring during the two LOS (line-of-sight) periods at the beginning of the flight and the end of the first orbit/beginning of the second, were returned.

\subsection{Recovery}

As a result of the flight termination occurring very late in the Antarctic season, and the long distance from McMurdo, we were not able to recover the instrument during the same season. The recovery was carried out in the 2014/2015 Austral Summer, two years after the flight. Because of its remote location, recovery required setting up a field camp to disassemble the instrument and prepare components for transportation. On Dec. 28, 2014, one of our recovery team members, Thomas Hams, arrived at the recovery site with the grooming team who prepared the skiway for the Basler aircraft. The instrument had been sunk under the snow and needed to be dug up. He spent about a week digging the instrument from the snow (Fig.Ђ).

The instrument had been turned upside down and rested on top of the rotator. The upper most scintillation detector of one module was damaged by the rotator. The other detectors did not appear to be damaged. All HV cables and signal cables were carefully disconnected from the PMTs (Fig.6), then the detectors were unstacked from the support structure. On Jan. 24, 2015, the Basler aircraft arrived at the recovery site with the rest of the recovery team members, Sean Fitzsimmons and Ryan Murphy. All of the detectors, electronics, and instrument support components were loaded into the Basler aircraft. The detecter components were designed to be recoverable with either the Basler or Twin-Otter aircraft, but the Basler aircraft was preferred since it allows full detector layers to be directly loaded, with the least potential for damage in transport. The Basler flew the instrument to the South Pole, then the instrument was loaded onto Air Force pallets and 


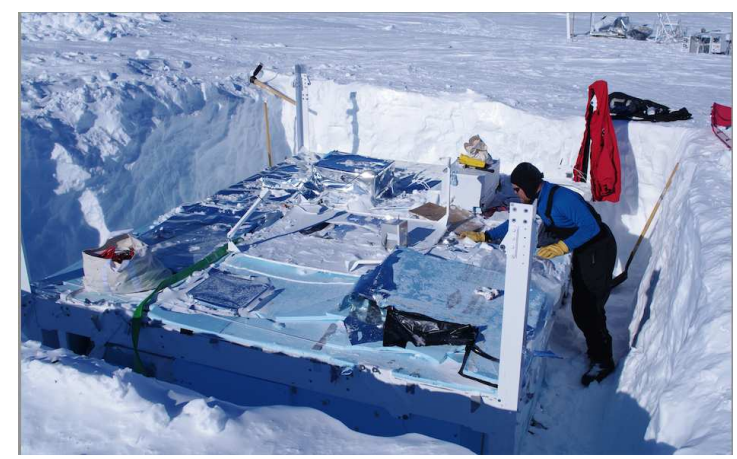

Figure 5: Photo 1 of the recovery site. The SuperTIGER-1 instrument was buried under the snow.

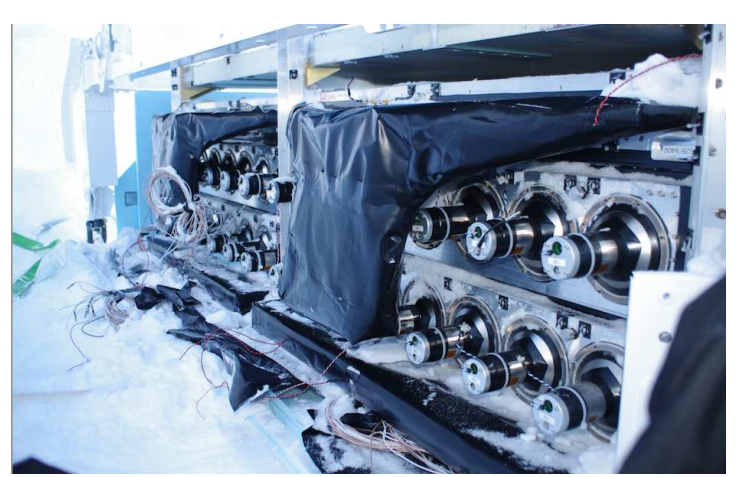

Figure 6: Photo 2 of the recovery site. The HV cables and signal cables were carefully removed from the PMTs.

transported to McMurdo by LC130. The instrument was loaded into the shipping container at McMurdo and shipped back to the US.

\section{Data Analysis}

Our analysis has so far been focused on the TDRSS data and LOS data at the moment. The healthiness of each packet in the telemetry data has been examined by the checksum. We took pedestal data every 20 minutes during the flight and performed gain calibrations using Fe events about every 2 hours depending on the data transmission rates. The position-dependent response (mapping) for each detector was produced on a $2 \mathrm{~cm} \times 2 \mathrm{~cm}$ grid using Fe events from the full flight duration. In addition to the regular position map, four new response maps were produced. The detector response depends on not only the position but also the particle angle. The angle dependence of course depends on position, so we needed to produce two-dimensional (position, angle) response maps to get an ideal response map. However, the number of Fe events observed was limited and not enough to produce a statistically meaningful two-dimensional response map. Instead, we assumed that the angle dependence came from the distortion of the detector and characterized the distortion in four motions (two gradients and two twists). The distortion maps were produced on a $4 \mathrm{~cm} \times 4 \mathrm{~cm}$ grid using Fe events from the full fight duration. The position map and the 4-distortion maps do correct the position and angle dependence of the detectors.

Particle charge is measured using both [dE/dx vs. CK] and [high-n-CK vs. low-n-CK] techniques. Angles of incidence and instrument response are corrected using particle trajectories measured by the hodoscopes. For low-energy events, below the low-index ( $n=1.043$ and $n=1.025)$ aerogel CK (C0) thresholds and extending slightly above the $\mathrm{C} 0$ threshold, the sum of two scintillator signals $(\mathrm{ST}=\mathrm{S} 1+\mathrm{S} 2)$, with a small velocity correction from the high-index $(\mathrm{n}=1.49)$ acrylic CK (C1) signal, is used to determine Z. Our data are shown in Fig.Z, which is a scatter plot of $\mathrm{S} 1$ vs $\mathrm{C} 1$ in one module. The charge bands are clearly visible up to Ni. The S3 signal helps identify and reject nuclei interacting in the instrument but is not used in the primary charge measurement. For high-energy events, above the $\mathrm{C} 0$ threshold, the $\mathrm{C} 1$ signal, with a small correction from the $\mathrm{C} 0$ signal, is used to measure charge. These data are shown in Fig. 8 , which is a scatter plot of $\mathrm{C} 1$ vs $\mathrm{C} 0$ 


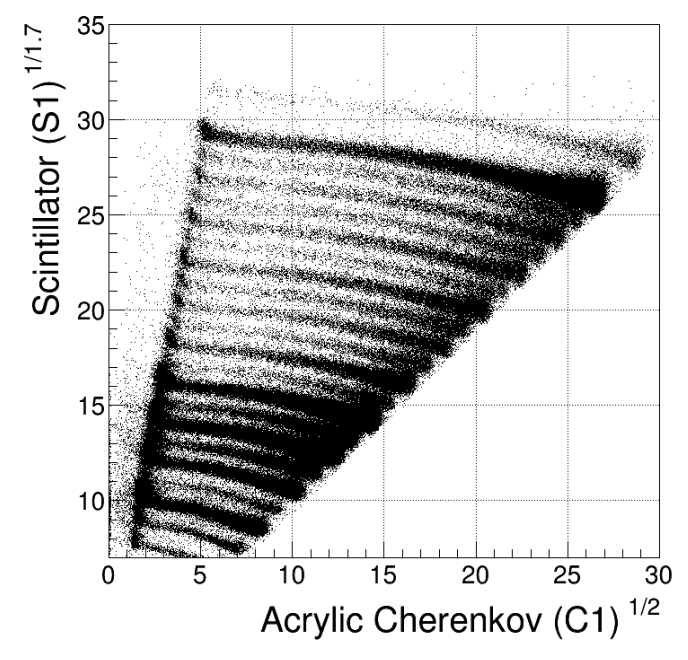

Figure 7: Cross-plots of Super-TIGER data for scintillation detector (S1) vs acrylic Cherenkov (C1).

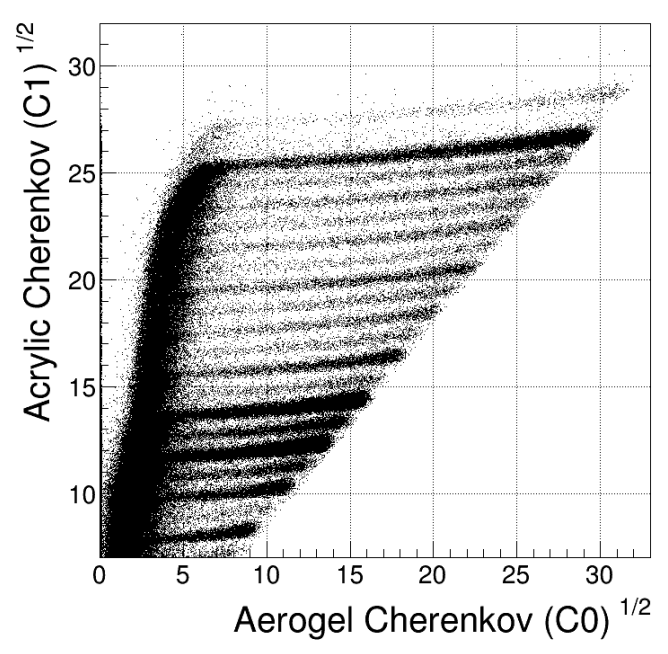

Figure 8: Cross-plots of Super-TIGER data for acrylic Cherenkov (C1) vs aerogel Cherenkov (C0).

in one module. Again, the charge bands are clearly visible. Although the underlying measurement technique at low energies is $[\mathrm{dE} / \mathrm{dx}$ vs $\mathrm{CK}]$, the use of organic scintillators means that the technique is actually $[\mathrm{dL} / \mathrm{dx}$ vs $\mathrm{CK}]$, where $\mathrm{L}$ is the light produced by particle energy loss in the scintillator material. Although $\mathrm{dE} / \mathrm{dx} \propto Z^{2}, \mathrm{~L}$ exhibits saturation effects at high specific $\mathrm{dE} / \mathrm{dx}$ [Q] , and the scintillator response becomes more complicated. However, the $\mathrm{Z}$ resolution $\sigma_{Z} \leq 0.25$ needed to resolve the relatively rare odd- $Z$ elements can still be achieved by correcting the non- $Z^{2}$ behavior of the scintillators for velocity and particle charge dependence. The Gaussian fit to the Fe peak has $\sigma_{Z}=0.15$ c.u. for the low-energy events (Fig.QI) and $\sigma_{z}=0.16$ c.u. for the high-energy-events (Fig.

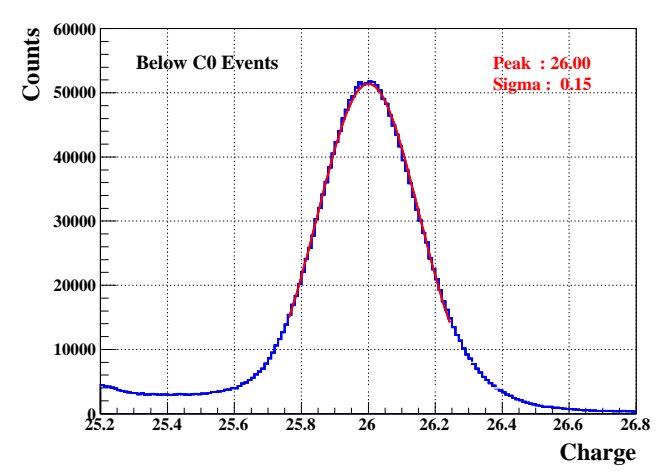

Figure 9: Charge histogram around $Z=26$ for lower enegy events.

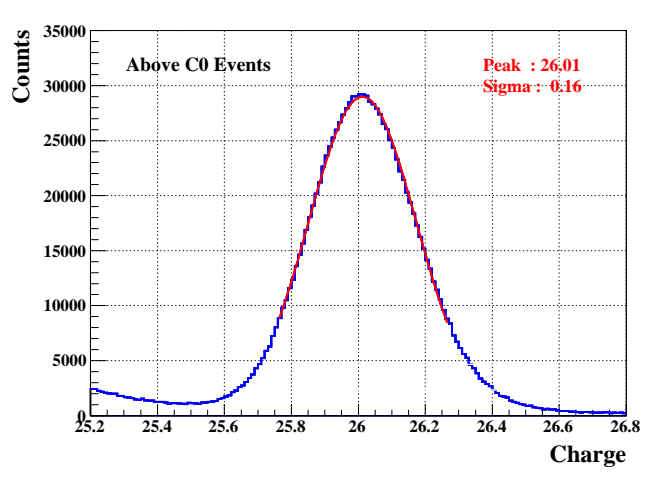

Figure 10: Charge histogram around $Z=26$ for higher enegy events.

The SuperTIGER-1 measurements are discussed in [ [0, $\square]$ and in [ㅈ] with the Cosmic Ray 
Isotope Spectrometer (CRIS) on the NASA Advanced Composition Explorer (ACE) satellite measurements in the context of their stringent tests of the $\mathrm{OB}$ association model for the origin of galactic cosmic rays.

\section{Future Plan}

As noted in the previous section, we successfully recovered the instrument from Antarctica. We are now evaluating the condition of each detector in detail. The detectors have kept their outer shape, and the radiators didn't have visible cracks at first glance; however, we have found some of the PMTs were damaged either on landing or by two years storage time in the snow. Depending on the results of the evaluation, we will rebuild some detector components and integrate the instrument for the SuperTIGER-2 flight planned for December 2017.

Acknowledgment: The SuperTIGER program is supported by NASA. We wish to thank the Columbia Scientific Balloon Facility (CSBF) personnel and the Wallops Balloon Program Office (BPO) for their excellent efforts that resulted in our highly successful long-duration balloon flight. We also wish to thank the National Science Foundation Office of Polar Programs for their outstanding logistical support which made this investigation possible.

\section{References}

[1] B. F. Rauch et al., Cosmic Ray origin in OB Associations and Preferential Acceleration of Refractory Elements: Evidence from Abundances of Elements ${ }_{26} \mathrm{Fe}$ through ${ }_{34} \mathrm{Se}$, ApJ 697 (June, 2009) 2083-2088, [arXiv:0906.2027].

[2] J. C. Higdon and R. E. Lingenfelter, The Superbubble Origin of ${ }^{22}$ Ne in Cosmic Rays, ApJ 590 (June, 2003) 822-832.

[3] W. R. Binns et al., Cosmic-Ray Neon, Wolf-Rayet Stars, and the Superbubble Origin of Galactic Cosmic Rays, ApJ 634 (Nov., 2005) 351-364, [astro-ph/0508398].

[4] W. R. Binns et al., Constraints on Galactic Cosmic-Ray Origins from Elemental and Isotopic Composition Measurements, in 33rd International Cosmic Ray Conf. (Rio de Janeiro) 0646 (2013).

[5] H. S. Ahn et al., Measurements of the Relative Abundances of High-energy Cosmic-ray Nuclei in the TeV/Nucleon Region, ApJ 715 (June, 2010) 1400-1407.

[6] R. P. Murphy et al., Abundances of Ultra-Heavy Galactic Cosmic Rays from the SuperTIGER Instrument, in Proceedings of ICRC2015 (2015).

[7] A. W. Labrador et al., Galactic Composition and Spectra for Ne through Ni from 0.8 to $10 \mathrm{GeV/nuc}$ with the SuperTIGER Instrument, in Proceedings of ICRC2015 (2015).

[8] W. R. Binns et al., The SUPERTIGER Instrument: Measurement of Elemental Abundances of Ultra-Heavy Galactic Cosmic Rays, ApJ 788 (June, 2014) 18.

[9] M. H. Salamon and S. P. Ahlen, Plastic scintillator response to relativistic Ne, Ar, Fe Ions, Nuclear Instruments and Methods in Physics Research 195 (1982) 557-568.

[10] T. Hams et al., Elemental Abundances of Ultra-Heavy GCRs measured by SuperTIGER and ACE-CRIS and the Origin of Galactic Cosmic Rays, in Proceedings of ICRC2015 (2015). 